\title{
Relevé, analyse et publication de données concernant la qualité des traitements médicaux
}

\begin{abstract}
Version abrégée des recommandations La qualité des traitements médicaux est difficile à définir et à examiner. On peut distinguer trois dimensions: en premier lieu, la qualité structurelle (par ex. le nombre de soignants par patient, la maintenance appropriée des équipements techniques); en deuxième lieu, la qualité du processus (par ex. le traitement d'une maladie d'après l'état actuel des connaissances); en troisième lieu, la qualité du résultat-ou outcome - (par ex. le taux de guérison, de réhospitalisations non planifiées, la satisfaction des patients, le taux de mortalité). Chacune de ces dimensions possède des avantages et des inconvénients spécifiques auxquels il faut prêter attention.
\end{abstract}

La mesure et la saisie de la qualité des traitements médicaux ainsi que la comparaison des différents fournisseurs de prestations doivent être effectuées d'une manière d'autant plus soigneuse qu'elles sont coûteuses, complexes sur le plan méthodique et sujettes à erreurs. Elles ne devraient pas non plus être exposées à une compétition pour la publication la plus rapide ou celle qui fait les plus gros titres.

La publication de données concernant la qualité doit permettre d'atteindre plusieurs objectifs. Chez les fournisseurs de prestations dont la qualité est médiocre, les données doivent entraîner des mesures améliorant la qualité; les fournisseurs qui offrent, quant à eux, une bonne qualité se voient confirmés dans leur travail. La publication permet à tous les patients de recevoir les informations nécessaires, d'une part pour choisir des fournisseurs de prestations qui proposent une bonne qualité et, d'autre part pour être informé au sujet des prestataires dont la qualité est insuffisante. L'objectif est de stimuler la concurrence entre les prestataires, et de créer des bases de données, pour pouvoir récompenser de manière appropriée la bonne qualité des traitements médicaux.

La garantie de la qualité des traitements médicaux est inscrite depuis 1996 dans la Loi sur l'assurance-maladie. Celle-ci exige que les fournisseurs de prestations ou leurs organisations élaborent des concepts et des pro- grammes concernant les exigences envers la qualité des prestations et la promotion de la qualité. En outre, un nouvel article 22a LAMal est en vigueur depuis le $1^{\text {er }}$ janvier 2009: il oblige les fournisseurs de prestations «à communiquer aux autorités fédérales compétentes les données qui sont nécessaires pour surveiller l'application des dispositions de la présente loi relatives [...] à la qualité des prestations».

Les patients, les politiciens, les assurances maladie et les fournisseurs de prestations manifestent tous un intérêt marqué et compréhensible pour une qualité élevée des traitements médicaux. C'est pourquoi il faut saluer et encourager d'une manière générale la publication de données dont le but est l'évaluation de cette qualité. Le public est en droit d'être informé au sujet de la qualité des prestations médicales. Mais au-delà des effets escomptés (amélioration de la qualité, transparence et possibilité de choix pour les patients), il faut également prendre en considération les effets indésirables, tels que les informations incorrectes ou les décisions erronées qui résultent de données mal recueillies et/ou analysées de manière inexacte.

Les recommandations du groupe de travail de l'ASSM qui a été chargé de cette question établissent les conditions préalables à considérer lors de la publication de données concernant la qualité. La plus importante est le respect des critères suivants qui sont récapitulés dans une liste de contrôle et qui fournissent la garantie que les données relevées sont à la fois pertinentes, justes et intelligibles.

\section{Pertinence}

- Les indicateurs choisis représentent-ils la qualité des traitements médicaux?

- L'indicateur considéré concerne-t-il la structure, un processus ou le résultat d'un traitement?

- Le responsable du choix des indicateurs est-il mentionné?

\section{Justesse}

- Les indicateurs sont-ils définis de manière précise?
- La totalité des patients chez qui l'indicateur est mesuré, est-elle clairement décrite?

- Existe-t-il des indications concrètes sur le nombre de patients chez lesquels l'indicateur a été relevé?

- Dans le cas d'une enquête auprès des patients, existe-t-il des indications sur le taux de réponse?

- Les instruments servant à la mesure des indicateurs sont-ils valides et fiables?

- Des indications sur le personnel qui a recueilli les données sont-elles mentionnées dans le rapport?

- La source des données est-elle décrite?

- La durée de la mesure et de l'intervalle de temps est-elle indiquée?

- Les données nécessaires à une éventuelle rectification de la composition du collectif des patients (casemix) ont-elles été recueillies?

- La qualité des données relevées a-t-elle été contrôlée?

- Y-a-t-il des indications sur la manière dont on a procédé avec les données manquantes?

- La présentation des résultats est-elle adéquate et intelligible?

- La réalisation de la comparaison avec d'autres fournisseurs de prestations est-elle décrite de manière détaillée?

\section{Intelligibilité}

- Les résultats sont-ils présentés de façon intelligible et interprétés de manière compréhensible?

Chaque rapport publié concernant la qualité devrait inclure la liste de contrôle remplie au sens d'une déclaration. À moyen terme, «l'organisation nationale pour l'assurance-qualité et la sécurité des patients» (encore à créer et déjà réclamée par une initiative parlementaire) aura pour mission d'exiger de chaque fournisseur de prestations, la publication de cette liste de contrôle (ou d'une liste semblable) et le respect de ses critères. Par ailleurs, les médias seront tenus d'indiquer, lors du compte rendu, si les rapports cités concernant 
la qualité se servent de la liste de contrôle et dans quelle mesure ils remplissent ses exigences.

\section{Introduction}

\section{Contexte}

Les patients, les politiciens, les assurances maladie et les fournisseurs de prestations ${ }^{2}$ manifestent un intérêt marqué et légitime pour une qualité élevée des traitements médicaux. Une entreprise privée et quelques hôpitaux ont publié en 2007 des données concernant la qualité des traitements, après plusieurs années caractérisées par une activité réduite - ou en tout cas imperceptible par le public - en matière de garantie de la qualité. D'autres institutions et organisations annoncèrent à l'époque une prochaine publication de données concernant la qualité. Les réactions furent très variées parmi le public: certains saluèrent le fait que des données soient enfin publiées sur la qualité des traitements, créant ainsi davantage de transparence; d'autres émirent publiquement des critiques sévères sur la manière dont les données avaient été relevées et interprétées. Mais on salua, d'une manière générale, la nécessité d'une amélioration de la transparence en ce qui concerne la qualité des prestations fournies dans le système de santé; cet avis est partagé par l'Académie Suisse des Sciences Médicales (ASSM).

Outre les conséquences souhaitées d'une transparence accrue, les effets négatifs et potentiellement indésirables de la publication de données demandent également réflexion: il est ici plus particulièrement question de la publication de données erronées et/ou difficilement interprétables. Suite à la critique substantielle adressée à la façon dont les données publiées jusqu'ici furent relevées et publiées, l'ASSM a mis en place un groupe de travail et l'a chargé de:

1. la création de standards (ou critères) qui doivent être respectés lors de la publication $^{3}$ de données concernant la qualité des prestations médicales (données concernant la fourniture de prestations);

2. l'énumération de fondements juridiques et de principes éthiques à considérer lors de la publication de données concernant la qualité.

Dans le cadre de sa réflexion, le groupe de travail a constaté qu'une limitation à la seule «publication» de données ne serait pas concluante, le relevé et l'analyse exacts des données représentant une condition préalable et impérative à leur publication. Un devoir de diligence et un sens des responsabilités particuliers s'imposent, car la publication de telles données - en particulier, dans les médias - débordera le cercle étroit des experts. La population générale n'est pas en mesure d'apprécier à sa juste valeur le potentiel ou/et les limitations de telles données et a besoin qu'elles soient correctes, pertinentes et intelligibles. Sinon, le risque est réel que l'on tire des conclusions erronées qui auront des conséquences négatives, tant pour les patients que pour les fournisseurs de prestations.

Le présent document ne se limite donc pas simplement à émettre des recommandations sur la publication de données concernant la qualité des traitements médicaux, mais énonce également des critères à respecter lors du relevé et de l'analyse de ces données. Les recommandations s'adressent ici bien évidemment à différents groupes cibles.

L'ASSM a conscience du fait que les présentes recommandations ne représentent qu'un pas vers une garantie durable et une amélioration de la qualité des traitements médicaux. Les recommandations s'entendent comme une incitation à mettre en pratique cet objectif primordial avec tout le soin nécessaire, et non à le discréditer par des classements ou à l'assimiler à une opération de promotion. D'autres mesures seront évidemment nécessaires, et en particulier l'élaboration de manuels. D'après l'ASSM, d'autres institutions et organisations sont cependant mieux adaptées pour assumer cette mission-là.

\section{Objectifs et conséquences de la publication de données concernant la qualité}

La publication de données concernant la qualité doit permettre d'atteindre plusieurs objectifs. Lorsqu'elle concerne la qualité des traitements, la publication de données a pour but d'instaurer la transparence. Chez les fournisseurs de prestations dont la qualité est médiocre, les données doivent entraîner des mesures améliorant la qualité; les fournisseurs qui offrent, quant à eux, une bonne qualité se voient confirmés dans leur travail $[1,2]$. La publication permet à toutes les parties prenantes de recevoir les informations nécessaires, d'une part pour choisir des fournisseurs de prestations qui proposent une bonne qualité et, d'autre part pour être informé au sujet des prestataires dont la qualité est insuffisante. L'objectif est de stimuler la concurrence entre les prestataires, et de créer des bases de données, pour pouvoir récompenser de manière appropriée la bonne qualité des traitements médicaux.
Outre ces conséquences prévues de la publication de données, il faut également tenir compte des effets indésirables qui proviennent surtout de la publication de données erronées. Les patients, fournisseurs de prestations et prestataires financiers (assurances maladie et cantons) peuvent être incités à tirer des conclusions erronées, lorsque la saisie des données est médiocre, entraînant éventuellement la publication de fausses indications. Le risque existe de voir des patients induits en erreur choisir, de ce fait, des fournisseurs de prestations qui offrent une qualité inférieure. Lorsque les données publiées sont meilleures que la qualité réellement fournie, les mesures nécessaires d'amélioration de la qualité sont négligées. En revanche, lorsque la qualité produite est vraiment meilleure que celle qui est restituée par les données publiées, la bonne réputation du fournisseur de prestations peut en souffrir et des ressources seront potentiellement investies dans des activités inutiles. En faisant dépendre le montant du paiement des prestations médicales de la qualité fournie («rémunération au rendement» ou pay for performance) [3], le risque existe de récompenser les faux prestataires et de répartir les ressources de manière déloyale. Les fournisseurs de prestations modifient dans la foulée leurs priorités et la publication de chiffres «à l'aspect flatteur» l'emporte sur des activités visant à une amélioration de la qualité [4].

La sélection des risques est l'une des conséquences possibles qui ont déjà été observées à l'étranger. Cela revient à dire que les hôpitaux pourraient en venir soit à ne pas accueillir les patients qui présentent un risque accru, soit à les adresser rapidement à d'autres cliniques dans le but de maintenir leurs propres données concernant la qualité au niveau le plus élevé possible.

\section{Les différentes formes de qualité des traitements médicaux}

La qualité des traitements médicaux est une notion difficile à définir. La quantification et l'évaluation de la qualité représentent, de ce fait, des défis majeurs [5]. Donabedian [6] a défini le concept de la qualité des traitements médicaux comme «une propriété des soins aux patients qui peut varier dans son extension». Cette définition ne concerne évidemment pas uniquement les activités des médecins, mais inclut également celles des infirmiers, des physiothérapeutes, des ergothérapeutes et d'autres professionnels de la santé. De la même manière, la mesure de la qualité ne se limite pas au domaine hospitalier, mais comprend également le secteur ambulatoire. 
La qualité médicale peut être décrite par trois dimensions: en premier lieu, la qualité structurelle (par ex. le nombre de soignants par patient, la maintenance appropriée des équipements techniques); en deuxième lieu, la qualité $d u$ processus (par ex. le traitement d'une maladie d'après l'état actuel des connaissances); en troisième lieu, la qualité du résultat-ou outcome - (par ex. le taux de guérison, de réhospitalisations non planifiées, la satisfaction des patients, le taux de mortalité). Il n'est pas possible de considérer ces trois dimensions indépendamment les unes des autres. Des structures appropriées sont nécessaires pour offrir une bonne qualité du processus qui déterminera, dans des proportions plus ou moins importantes, la qualité du résultat. La qualité est quantifiée par ce que l'on appelle des «indicateurs de qualité» et le «degré» de qualité est décrit par une comparaison avec une norme fixée ou d'autres fournisseurs de prestations.

Les présentes recommandations se penchent principalement sur la qualité du processus et la qualité du résultat. La qualité structurelle est une condition préalable importante, mais insuffisante pour obtenir une bonne qualité des traitements et du résultat. Les comptes rendus concernant la qualité structurelle doivent également être pertinents, justes et intelligibles. Le débat n'est pas clos, pour définir quelles situations nécessitent la mesure de la qualité du processus et quelles situations exigent la mesure de la qualité du résultat. Les deux méthodes présentent des avantages et des inconvénients et la décision en faveur de l'une ou l'autre qualité dépendra du contexte.

\subsection{Qualité structurelle}

La «structure» d'un prestataire est décrite à l'aide de chiffres clés et comprend les personnes, les moyens et les ressources dont dispose le fournisseur de prestations pris isolément; de plus, elle comporte des indications sur l'organisation de l'institution. Si une «structure» adéquate représente la condition préalable à l'obtention d'une qualité élevée du processus et du résultat (ou outcome), elle n'est toutefois pas, prise séparément, la garante d'une bonne qualité des traitements. Il est impossible de se prononcer sur la qualité structurelle, sur la seule base de chiffres clés, tels que, par exemple, le nombre de médecins et d'infirmiers; des indications concernant le niveau de formation des médecins, des infirmiers, des thérapeutes et du personnel médical sont également nécessaires pour estimer cette qualité structurelle.
Avantages de la mesure de la qualité structurelle

Les chiffres clés tels que le nombre de soignants par patient ou le nombre de cas par maladie sont en règle générale relativement faciles à relever. La comparabilité entre différents fournisseurs de prestations est également possible.

\section{Inconvénients et limites de la mesure de la qualité structurelle}

Les chiffres clés ne permettent, à eux seuls, que des déclarations limitées concernant la qualité des traitements médicaux (cela vaut, du reste, pour toutes les dimensions de la qualité). Pour donner un exemple: un nombre suffisant de médecins est nécessaire pour obtenir un traitement adéquat des patients. Mais le nombre seul ne suffit pas pour évaluer, par exemple, le taux de réussite de mesures de réanimation. Un entraînement régulier du personnel médical est également nécessaire pour cela.

\subsection{Qualité du processus}

\section{(incluant la qualité de l'indication ${ }^{4}$ )}

La première mission du traitement médical consiste à faire ce qui est considéré comme nécessaire par les experts correspondants et dans l'état actuel des connaissances. La qualité du processus relève combien de fois les mesures considérées comme nécessaires par les experts furent prises au cours du traitement. À titre d'exemple et sauf contre-indication, tous les patients devraient bénéficier d'une prophylaxie de la thrombose après une intervention orthopédique; d'autre part, tous les patients victimes d'un infarctus du myocarde devraient recevoir de l'aspirine. L'apparition d'une thrombose postopératoire et le risque d'un autre infarctus du myocarde sont ainsi réduits.

\section{Avantages de la mesure de la qualité du processus}

Les indicateurs sont faciles à fixer et la composition du collectif des patients (casemix) est sans grande importance lors de l'interprétation des résultats. Les indicateurs qui permettent d'évaluer si les mesures introduites correspondent bien à l'état actuel des connaissances sont, en règle générale, faciles à fixer. Lorsque les experts auront défini les mesures adéquates, les unités servant à leur évaluation pourront en être déduites.

Que signifie la notion de composition du collectif des patients (casemix)? Les patients d'un hôpital ou d'un médecin praticien qui sont atteints d'une certaine maladie, ne représentent pas un collectif homogène, en ce qui concerne le pronostic. Un collectif de patients est pratiquement toujours composé de patients aux pronostics variés. Dans un même hôpital, on soignera des patients atteints d'un petit infarctus sans gravité et des patients souffrant d'un infarctus étendu qui affecte gravement la fonction cardiaque. Indépendamment de la thérapie, le pronostic des patients victimes d'un petit infarctus sera meilleur que celui des patients souffrant d'un infarctus très étendu. Le fait que les patients soient différents face au pronostic est appelé la composition du collectif des patients (casemix). En règle générale, un nombre plus élevé de patients au pronostic moins favorable est pris en charge dans des hôpitaux hautement spécialisés.

Exemple: les patients doivent recevoir de l'aspirine, indépendamment du degré de gravité de l'infarctus du myocarde et d'autres maladies éventuellement associées. Il est possible de saisir et de documenter séparément les cas des patients auxquels il n'est ni possible ni souhaitable de prescrire de l'aspirine, pour quelque raison que ce soit.

La composition variée des collectifs de patients - c'est-à-dire le casemix - n'a, en règle générale, pas une grande influence sur les résultats de la qualité du processus; une comparaison - avec d'autres fournisseurs de prestations ou avec une valeur de référence fixée (benchmark) - est ainsi plus facile que dans le cas des résultats portant sur la qualité du résultat (outcome).

\section{Inconvénients et limites des mesures}

de la qualité du processus

Seule une partie de la qualité totale est recueillie et d'autres décisions cliniques - qui appartiennent également à un traitement adéquat ne sont, pour cette raison, pas saisies. Dès lors que seuls un ou deux aspects sont enregistrés pour caractériser un tableau clinique, les médecins et les autres professions médicales ont, lors du traitement, avant tout tendance à faire attention à [7] ces points («l'attention portée au test» ou care to the test) et peut-être à négliger d'autres aspects.

Les patients ne s'intéressent pas, en premier lieu, aux indications concernant la qualité du processus. Ils partent du principe que l'on fait le nécessaire. Pour le patient, c'est le résultat des soins - dans leur globalité - qui compte et qui peut être quantifié à l'aide d'indicateurs qui reflètent le résultat (outcome).

\subsection{Qualité du résultat}

Le traitement médical a pour objectif l'amélioration de l'état de santé des patients, c'està-dire une amélioration de la qualité de vie et une réduction de la morbidité et/ou de la mortalité. La qualité de vie et les taux de morbi- 
dité et de mortalité sont des exemples d'indicateurs qui mesurent la qualité du résultat (ou de l'outcome). Chez le patient - pris isolément et au sein du collectif -, le résultat dépendra de différents facteurs: d'une part, du traitement médical et donc de la qualité du processus; d'autre part, de la composition du collectif des patients (casemix) et d'autres facteurs aléatoires impossibles à décrire plus en détail et, par conséquent, non mesurables [8]. Cela revient à dire que le résultat dépendra de facteurs qui ne peuvent pas être relevés avec précision.

\section{Avantages des mesures de la qualité du résultat}

La qualité du résultat mesure ce qui constitue, en règle générale, la priorité la plus élevée pour le patient. Celui-ci attend de guérir, avec la probabilité la plus élevée possible ou au moins de voir ses douleurs soulagées. Pour le patient, c'est en premier lieu le résultat du traitement qui compte et moins la manière dont il a été appliqué. Outre le résultat médical, c'est la satisfaction du patient à l'égard du traitement qui constitue un paramètre pertinent auquel on aura recours pour évaluer un fournisseur de prestations.

\section{Inconvénients et limites des mesures de la qualité du résultat}

Aussi souhaitable que puisse être la mesure de ces paramètres, l'interprétation de la qualité du résultat est difficile à fournir. Cette difficulté provient de la composition du collectif des patients (casemix) qui influence les résultats des mesures portant sur le résultat (outcome).

Exemple: un collectif de patients souffrant d'un petit infarctus simple obtient un meilleur pronostic (mesuré en taux de survie) qu'un collectif de patients atteints d'un infarctus étendu et de troubles du rythme cardiaque qui représentent un danger majeur. Bien que les deux collectifs soient traités et soignés dans les règles de l'art médical, les résultats peuvent, malgré tout, être différents pour les deux collectifs.

Dès lors, on tentera, à l'aide de méthodes statistiques, de compenser les différences qui existent au sein de la composition du collectif des patients (casemix), afin de pouvoir comparer la qualité du résultat entre différents prestataires (tels que des hôpitaux). Les facteurs décisifs concernant le pronostic des patients étant très souvent inconnus, aucune correction statistique ne peut être réalisée. Il n'existe, à l'heure actuelle, aucune méthode absolument sûre et robuste qui permettrait de résoudre ce problème fondamental [8]. Les comparaisons qui portent sur les résultats entre les différents hôpitaux doivent, par conséquent, être interprétées avec beaucoup de prudence. Le nombre faible de cas entraîne une limitation supplémentaire à la mesure du résultat et à la comparabilité entre les fournisseurs de prestations. Les effectifs des patients doivent être relativement élevés pour permettre la constatation - avec une probabilité importante - d'une amélioration ou d'une détérioration de la qualité du résultat, au sein du même hôpital ou entre différents hôpitaux (et en admettant que le problème de la composition du collectif des patients soit résolu de manière satisfaisante). Dans la plupart des hôpitaux suisses, les effectifs des patients sont trop faibles pour permettre la comparaison de nombreux résultats; une comparaison pertinente n'est donc possible qu'avec de grandes réserves.

\section{Aspects éthiques}

Les données concernant la qualité des traitements médicaux devraient être recueillies, analysées et communiquées de façon à ce que personne ne subisse de préjudice injustifié. Lors du relevé et de la publication de données concernant la qualité, deux aspects prennent une importance particulière d'un point de vue éthique. Premièrement, les données publiées et l'interprétation des données devraient être correctes. La publication de données incorrectes ou l'interprétation trompeuse sont inacceptables sur le plan éthique [9]. Deuxièmement, les projets concernant la garantie de la qualité nécessitent l'accord d'une commission d'éthique, aux termes de la «commission d'experts du secret professionnel en matière de recherche médicale». Dans son rapport, cette commission fait observer de manière explicite que «les études réalisées en interne concernant le contrôle de qualité» sont considérées comme «une recherche interne à l'entreprise» et par conséquent soumises à autorisation ${ }^{5}$. Cette déclaration fait l'objet d'une controverse et d'une interprétation variable selon les cantons. Il est donc recommandé de prendre contact avec les responsables cantonaux correspondants avant d'effectuer des mesures portant sur la qualité.

Un autre point crucial qui est discuté dans le contexte de la publication de données concernant la qualité et qui gagnera peut-être en importance dans le futur, concerne le devoir d'information des patients par le médecin traitant [10]. Lorsque les données concernant la qualité de différents hôpitaux sont publiées et que celles qui concernent par exemple le traitement des attaques d'apoplexie varient, la question se pose de savoir, si l'information aux patients à ce sujet fait partie des devoirs du médecin traitant: un argument de plus qui plaide en faveur de la nécessité de données correctes, lorsqu'elles sont publiées.

Comme nous l'avons déjà décrit, une stricte sélection des patients pourrait être l'une des conséquences de la publication de données concernant la qualité. En cas de sélection des risques, les patients «inintéressants» s'exposent à être poussés d'un hôpital à l'autre et éventuellement à ne pas recevoir de traitement adéquat.

\section{Aspects juridiques}

\subsection{Loi sur l'assurance-maladie ${ }^{6}$}

La garantie de la qualité des traitements médicaux est inscrite dans la LAMaL (article 58) et l'OAMaL (article 77) depuis 19967 . Elles exigent que les fournisseurs de prestations ou leurs organisations élaborent des concepts et des programmes en matière d'exigences de qualité des prestations et de promotion de la qualité. Les modalités d'exécution et leur financement doivent être réglés dans les conditions tarifaires ou dans des conventions particulières relatives à la garantie de la qualité conclues avec les assureurs ou leurs organisations. De plus, un nouvel article (art. 22a LAMaL) est en vigueur depuis le $1^{\text {er }}$ janvier 2009 qui oblige «les fournisseurs de prestations à communiquer aux autorités fédérales compétentes les données qui sont nécessaires pour surveiller l'application des dispositions de la présente loi relatives au caractère économique et à la qualité des prestations.» ${ }^{8}$ Conformement à la lettre $\mathrm{f}$, sont également inclus de manière explicite «les indicateurs de qualité médicaux». Les données recueillies par l'Office fédéral de la statistique (OFS) sont publiées par l'Office fédéral de la santé publique (OFSP) ${ }^{9}$.

Les publications officielles de données concernant la qualité dans le secteur de la santé doivent également respecter les règles du droit de la concurrence (voir 5.2.). Toutefois, les tiers (tels que les hôpitaux et les médecins) pourront vraisemblablement - lors de leurs activités publicitaires et d'information se référer à leur bonne foi en la justesse de la publication officielle ${ }^{10}$ (au moins tant qu'un débat scientifique, engagé par la suite, ne mène pas à la conclusion que la publication officielle est erronée ou au moins controversée, et que le tiers aurait dû, en y prêtant une attention raisonnable, avoir connaissance de ce débat). 


\subsection{Exigences en matière de loyauté, lors de la publication de données concernant la qualité et de leur utilisation par des tiers ${ }^{11}$}

La Loi fédérale contre la concurrence déloyale (LCD) ne s'applique pas seulement entre deux concurrents, mais également dans le cas d'allégations de la part de tiers concernant la concurrence, par exemple dans le contexte des comptes rendus publiés dans les médias ou des études scientifiques. La publication de données concernant la qualité dans le secteur de la santé doit, par conséquent, être en conformité avec les dispositions réglementaires sur la concurrence.

Selon la clause générale de l'article $2 \mathrm{LCD}$, est considéré comme déloyal «tout comportement (...) qui est trompeur ou qui contrevient de toute autre manière aux règles de la bonne foi». En outre, il convient notamment de respecter l'article 3, lettre a LCD, qui qualifie de déloyal le dénigrement par «des allégations inexactes, fallacieuses ou inutilement blessantes». La jurisprudence a souvent eu recours à cette disposition, dans le cadre de tests comparatifs de qualité et d'analyses comparatives financières, relatives à des offres de services.

La publication de données «inexactes» concernant la qualité est dans tous les cas déloyale. Il est par conséquent nécessaire de faire preuve de soin lors du relevé des données, par exemple en ce qui concerne la définition des indicateurs, les critères de sélection, la validité et la fiabilité des instruments de mesure, le contrôle de la qualité des données et la plausibilité des résultats. Il est inacceptable de passer outre des incertitudes qui seraient constatées lors du processus de traitement; il est nécessaire d'élucider les éventuelles sources d'erreurs. La description du relevé des données fournie dans ce rapport, satisfait aux principes du droit de la concurrence précédemment décrits; il est cependant nécessaire de garantir que les directives correspondantes seront également et intégralement appliquées dans la pratique.

Le domaine le plus délicat sur le plan juridique concerne la tromperie par le biais d'exemples. La jurisprudence a considéré, à plusieurs reprises, que même des indications exactes concernant un produit ou un service pouvaient induire en erreur; c'est notamment le cas lorsqu'elles produisent une impression erronée chez l'interlocuteur, du fait par exemple de particularités qui créent l'attente d'une qualité trop positive ou trop négative et contraire à la réalité. Dans le cas de descriptions comparatives, en particulier, il est nécessaire de veiller à ce que les indications se rap- portent à des services de même nature et de même catégorie ou à l'ensemble des concurrents qui présentent les mêmes qualités.

La jurisprudence applique un critère relativement exigeant au devoir de diligence, dans le contexte du respect des principes du droit de la concurrence. Ainsi, une description exacte des faits est requise dans la mesure où ils peuvent être constatés de manière objective; dans le cas de faits douteux, la présentation doit, si possible, être faite de manière à ce que le destinataire de l'information ait des indices suffisants pour se faire une opinion, en connaissance de cause. L'obligation de procéder à des clarifications supplémentaires inclut également l'exigence de rendre si possible plausibles les faits repris de tiers, d'écouter et de relater de manière pondérée des opinions réciproques et d'entreprendre sans idée préconçue le relevé et l'analyse de données concernant la qualité.

Il ne peut pas y avoir de complète sécurité juridique en matière de tromperie, rendue possible par le biais d'une transmission de l'information qui n'est pas parfaitement transparente. Toutefois, le meilleur critère reste le bon sens que l'auteur du relevé et de l'analyse de données concernant la qualité appliquera, en considérant la façon dont il peut et doit luimême comprendre les avis exprimés.

Il y a peu de chances que le critère des «allégations inutilement blessantes» soit pertinent dans le contexte des études scientifiques.

Enfin, il faut également attirer l'attention sur la nécessité de rendre, en règle générale, les données personnelles anonymes, sous peine d'infraction à la loi sur la protection des données.

\subsection{Droit de la publicité médicale} dans la Loi sur les professions médicales (LPMéd) et le code de déontologie de la FMH $^{6}$

La Loi sur les professions médicales (LPMéd) fait observer, dans l'article 40 consacré aux devoirs professionnels: «Les personnes exerçant une profession médicale universitaire à titre indépendant doivent observer les devoirs professionnels suivants: [...] d. s'abstenir de toute publicité qui n'est pas objective et qui ne répond pas à l'intérêt général; cette publicité ne doit en outre ni induire en erreur ni importuner.»

Sur le plan formel, cette directive ne s'applique qu'aux médecins qui dépendent de la LPMéd, excluant ainsi les hôpitaux. La jurisprudence montrera si cette disposition aura une influence - indirecte - sur la publicité des hôpitaux et des autres institutions où travaillent des médecins.
Le code de déontologie de la FMH et son annexe 2 (directives pour l'information et la publicité) reprennent pour l'essentiel, le concept de la LCD, tel qu'il a été décrit plus haut, dans la partie générale. Deux remarques complémentaires sont importantes:

a. Le code de déontologie de la FMH ne s'applique, du point de vue formel, qu'aux membres de la FMH. Cela signifie en particulier qu'il n'est pas directement applicable aux hôpitaux qui ne sont, somme toute, pas membres de la FMH. En revanche, selon l'article 20 alinéa 3 du code de déontologie, chaque membre de la FMH doit s'engager «à éviter qu'une publicité illicite soit faite en sa faveur par des tiers, de manière directe ou indirecte», autrement dit faire de la publicité qui serait interdite au membre lui-même (un devoir analogue n'a pas été inscrit dans l'article 40 LPMéd).

b. Contrairement à la LCD, le code déontologique de la FMH interdit la publicité avec des patients reconnaissants. Le médecin ne doit pas profiter du déséquilibre des forces qui existe entre lui et le patient, et recueillir ainsi des réactions positives de la part des patients qu'il pourrait exploiter dans la publicité.

\section{Recommandations}

Il faut saluer et encourager d'une manière générale la publication de données concernant la qualité. Le public est en droit d'être informé sur la qualité des prestations médicales. Mais au-delà des conséquences souhaitées - une transparence améliorée et une meilleure possibilité de choix pour les patients -, il est également nécessaire de prendre en considération les effets indésirables, c'est-à-dire les informations incorrectes et les décisions erronées qui résultent de données mal recueillies et/ou analysées de manière inexacte. La mesure et la saisie de la qualité des traitements médicaux doivent être effectuées d'une manière d'autant plus soigneuse qu'elles sont coûteuses, complexes sur le plan méthodique et sujettes à erreurs. Elles ne devraient pas non plus être exposées à une compétition pour la publication la plus rapide ou celle qui fait les plus gros titres.

Le débat entre les fournisseurs de prestations et l'apprentissage mutuel quant à la manière d'améliorer la qualité constituent les premiers objectifs de la mesure de la qualité. Mais pour cela, des structures et des incitations sont nécessaires afin de permettre et de soutenir ces processus. Selon l'ASSM, l'initiative parlementaire (07.486) concernant «l'organisation nationale pour l'assurance-qualité et la 
sécurité des patients» pose ici les exigences exactes: «Il faut fixer dans la législation les conditions nécessaires à la création d'une organisation nationale pour l'assurance-qualité et la sécurité des patients, qui mettra au point des instruments et des stratégies validés scientifiquement propres à garantir l'élaboration d'une politique nationale de la qualité dans le domaine de la santé, qui assurera la coordination et l'évaluation des stratégies et qui élaborera les bases d'un système transparent en termes de qualité des résultats et de sécurité des patients.»

Le groupe de travail esquisse ci-après les conditions préalables qui doivent, d'après lui, être remplies pour la publication de données concernant la qualité; il définit en outre les critères dont le respect garantit des données à la fois pertinentes, correctes et intelligibles.

Ces critères sont regroupés dans une «liste de contrôle». Le groupe de travail recommande que chaque rapport publié concernant la qualité contienne la liste de contrôle remplie. À moyen terme, les autorités compétentes auront pour mission d'exiger de chaque fournisseur de prestations, la publication de cette liste de contrôle (ou d'une liste semblable) et le respect des critères.

Les médias sont tenus d'indiquer, dans leur compte rendu, si les rapports cités concernant la qualité se servent de la liste de contrôle et dans quelle mesure ils remplissent ses exigences.

\section{Conditions préalables à la publication}

Les données doivent être saisies de manière soigneuse et les résultats publiés doivent représenter la qualité réelle des traitements ou au moins certaines parties pertinentes. La confiance de la population dans le système de santé se détériorerait, s'il s'avérait que les indications étaient incorrectes ou erronées.

Divers critères sont à respecter lors de la saisie des données, afin de limiter autant que possible la publication de données incorrectes. Il n'existe aucun argument plausible qui justifierait que l'on retienne a priori des critères différents pour la quantification de la qualité des traitements médicaux et pour les études concernant l'efficacité d'un médicament [11]. Dans les deux cas, lors de la mesure de la qualité des traitements et pendant le contrôle de l'efficacité de médicaments, des données incorrectes peuvent présenter des inconvénients pour les patients et les rendre inacceptables sur le plan éthique. La recherche concernant l'efficacité des interventions thérapeutiques possède une longue tradition et des critères clairs, élaborés au cours des décennies passées, doivent être remplis afin de rendre les résultats de la recherche dignes de confiance. Dans le domaine de la recherche (encore relativement récent) sur la mesure de la qualité, les bases méthodologiques ne sont pas encore aussi clairement définies que pour la recherche clinique. Plusieurs concepts et principes propres à cette dernière peuvent être repris ici et n'ont pas besoin d'être créés.

Les chiffres publiés doivent remplir trois critères généraux, étant donné que la publication des données concernant la qualité aura des conséquences - souhaitées et indésirables et que le financement des fournisseurs de prestations sera éventuellement rendu dépendant de la qualité des traitements fournis. Les chiffres publiés doivent être pertinents, justes et intelligibles pour le lecteur.

\section{Critères pour l'évaluation de données publiées concernant la qualité}

On trouvera ci-dessous des propositions de critères permettant d'évaluer la pertinence, la justesse et l'intelligibilité des données publiées concernant la qualité. Dans le cas idéal, un rapport remplira tous les critères, mais toutes les données publiées concernant la qualité ne rempliront pas l'ensemble des critères, entre autres pour des raisons de coût. Les indications concernant les critères remplis et non remplis viennent apporter de la clarté, donnent confiance et doivent permettre au lecteur une estimation des données publiées $[11,12]$.

\subsection{Pertinence}

\section{A. Les indicateurs choisis représentent-ils} la qualité des traitements médicaux?

On s'attend à ce que les données publiées et les rapports concernant la qualité (rapports Q) reflètent la qualité des traitements médicaux ou, au moins, plusieurs de ses aspects. C'est pourquoi le cadre conceptuel de la mesure de la qualité devrait être décrit dans l'introduction d'un rapport Q; de plus, il faudrait expliquer de manière plausible pourquoi et dans quelle mesure les indicateurs mesurés reflètent la qualité des traitements médicaux.

Exemples $^{12}$ : les infarctus du myocarde sont fréquents et représentent un problème clinique pertinent. Le risque d'un autre infarctus du myocarde peut être réduit par la prise de différents médicaments tels que l'aspirine. Les résultats de sondages et de contrôles aléatoires laissent supposer que les patients ne reçoivent pas tous ce médicament et qu'il existe de grandes différences entre les hôpitaux. Il se pourrait donc qu'il existe un sous-trai- tement des patients. C'est pourquoi la fréquence de prescription de l'aspirine est relevée dans les hôpitaux suisses, chez tous les patients victimes d'un infarctus du myocarde.

\section{B. L'indicateur considéré concerne-t-il} la structure, un processus ou le résultat d'un traitement?

L'indicateur est un paramètre qui permet de quantifier la qualité médicale. Le rapport devrait décrire s'il est question, dans ce cas, d'un indicateur de processus ou d'un indicateur de résultat. On choisira un indicateur de processus en supposant qu'une qualité élevée du processus mènera également à une amélioration du résultat (outcome) chez les patients (voir l'exemple ci-dessous). Cette hypothèse devrait être étayée par des indications publiées dans la littérature. Des informations concernant les limitations potentielles des indicateurs ainsi que les résultats de la mesure de la qualité sont souhaitables et facilitent l'intelligibilité des résultats.

Exemple: la mesure de la fréquence de prescription de l'aspirine chez les patients victimes d'un infarctus du myocarde est un indicateur de processus. Plusieurs études ont permis de démontrer que la prise régulière d'aspirine réduit le risque de subir un autre infarctus du myocarde. L'avantage de ce paramètre est d'être relativement simple à saisir. Son inconvénient est de ne saisir qu'un seul aspect $d u$ traitement au lieu du déroulement complet. Cela peut conduire à fixer l'attention principale sur la prescription d'aspirine et éventuellement à prêter moins d'attention à la prescription d'autres médicaments, également efficaces.

\section{Le responsable du choix des indicateurs est-il mentionné?}

Les indicateurs peuvent être sélectionnés par une personne seule, une clinique, une société spécialisée, des représentants de patients, une autorité politique, des assurances-maladie ou une équipe transdisciplinaire, en tenant compte des représentants de tous les partenaires impliqués. Une sélection par une commission multidisciplinaire est souhaitable et augmente l'adhésion aux mesures de la qualité.

Exemples: la mortalité hospitalière chez tous les patients souffrant d'un infarctus du myocarde, fut choisie par l'Office de la santé publique comme indicateur de la qualité. $L a$ "fréquence de réponse aux réclamations des patients» fut sélectionnée par $\mathrm{H+}$, en commun avec des représentants des organisations de patients, comme indicateur de la qualité. 


\subsection{Justesse}

A. Les indicateurs sont-ils définis de manière précise?

Les indicateurs devraient être décrits de manière précise et sans équivoque, afin d'empêcher des classifications incorrectes lors du relevé des données, et de permettre au lecteur du rapport Q de comprendre exactement ce qui a été mesuré. La définition exacte de l'indicateur revêt une importance toute particulière, lorsque différents fournisseurs de prestations sont comparés entre eux.

Il est tout aussi important de fixer clairement à quel moment l'information concernant les indicateurs est relevée. Il s'agit par exemple de savoir comment est évalué un décès qui survient, non pas à l'hôpital de premier recours, mais après le transfert à un autre hôpital ou à un service de soins.

Exemple 1: une infection postopératoire de la plaie (indicateur) peut être définie comme une rougeur des bords de la plaie, un écoulement purulent et des douleurs aux abords de la plaie. Une autre définition est la suivante: on est en présence d'une infection postopératoire de la plaie, lorsque la tomodensitométrie permet de déceler un abcès dans la région de la plaie. Des résultats différents peuvent être attendus en fonction de la définition choisie de l'infection de la plaie.

Exemple 2: le taux de mortalité est mesuré chez les patients victimes d'une attaque d'apoplexie, 30 jours après l'attaque. Le résultat a été mesuré chez tous les patients de l'hôpital A, 30 jours après l'attaque et considéré comme un décès à l'hôpital $A$.

\section{B. La totalité des patients chez qui l'indicateur} est mesuré, est-elle clairement décrite?

Pour certains projets concernant la qualité, on choisit les données de tous les patients d'un hôpital ou d'une clinique; pour d'autres projets, les données se limitent à une partie des patients. Il faudrait mentionner de façon détaillée comment cette sélection a eu lieu, dans pareil cas. Les résultats ne peuvent pas être interprétés - ou alors seulement sous toutes réserves - lorsque les critères de sélection ne sont pas clairs.

Exemple: à la sortie de l'hôpital, on a saisi si de l'aspirine avait été prescrite à tous les patients qui furent victimes d'un infarctus du myocarde. Une fois que les informations furent rassemblées chez 100 patients, choisis de manière aléatoire à partir d'un collectif de 334, les résultats ont permis des déductions sur le collectif tout entier. Le résultat ne peut toutefois pas être interprété lorsqu'un rapport indique que cette information a été recueil- lie chez 100 patients sur 334, sans mention explicite de la manière dont cette sélection a eu lieu.

\section{Existe-t-il des indications concrètes sur le nombre de patients chez lesquels l'indicateur a été relevé?}

Les rapports devraient contenir des indications relatives au nombre de patients chez qui l'indicateur a été relevé. Le nombre de patients revêt une importance particulière, lors de la comparaison de la qualité entre différents hôpitaux. Un nombre de cas trop faible rend impossible une comparaison interprétable entre différents fournisseurs de prestations. Avec un nombre réduit de cas, il n'est pas possible de distinguer si les variations dans les résultats obtenus, par exemple au niveau des taux de mortalité, reflètent vraiment une différence dans la qualité ou si elles sont fortuites. Des méthodes statistiques permettent de calculer le nombre de patients qu'il faut examiner pour parvenir à une conclusion pertinente.

Exemple: aux États-Unis, le taux de mortalité moyen suite à un remplacement de la hanche est de 0,3\%. Les résultats d'environ 2700 opérations seraient nécessaires pour prouver de manière sûre une multiplication par deux du taux de mortalité. Des nombres encore bien plus élevés sont nécessaires pour interpréter des modifications plus mineures du taux de mortalité comme une véritable détérioration ou une amélioration de la qualité. Les résultats d'au moins 200 opérations sont nécessaires pour prouver une multiplication par deux du taux de mortalité des pontages coronariens [13]. La plupart des hôpitaux n'atteignent pas, pour de nombreuses interventions chirurgicales, les chiffres nécessaires à une comparaison interprétable du taux de mortalité.

\section{Dans le cas d'une enquête auprès} des patients, existe-t-il des indications sur le taux de réponse?

Il est possible qu'une partie des patients ne puisse ou ne veuille pas donner de renseignements. Les sondages auxquels ne participent qu'un nombre réduit des personnes contactées produisent des résultats incertains. Il est impossible d'indiquer, de manière claire et universelle, combien de patients doivent participer à une enquête pour obtenir des résultats représentatifs. Le taux de participation le plus élevé possible est souhaitable.

Il se peut, dans d'autres situations également, que tous les patients ne soient pas saisis; dans ce cas, l'indication du taux de participation est également importante. C'est le seul moyen d'éviter ce que l'on appelle un «biais de sélection».
Exemple: les résultats sont difficiles à interpréter lorsque la satisfaction des patients est relevée à l'aide d'un questionnaire et que seuls $20 \%$ des patients remplissent ce questionnaire. Il est impossible de savoir si ce sont les patients plutôt insatisfaits ou les patients plutôt satisfaits qui ont répondu.

\section{E. Les instruments servant à la mesure}

des indicateurs sont-ils valides ${ }^{13}$ et fiables ${ }^{14}$ ? En principe, le relevé des données - concernant la prescription (on non) d'aspirine à la sortie de l'hôpital à un patient victime d'un infarctus du myocarde - est simple. En revanche, cela n'est pas le cas lors de la quantification de la satisfaction des patients. Des questionnaires spécialement conçus dans ce but sont utilisés pour saisir la satisfaction. En principe, il ne faudrait employer que des questionnaires validés et sûrs et citer les indications publiées à ce sujet dans la littérature. La décision doit être fondée de manière plausible, si aucun questionnaire déjà éprouvé n'est utilisé.

Exemple: le questionnaire PICKER a été utilisé pour la saisie de la satisfaction des patients. Des indications concernant la validité et la fiabilité sont accessibles en ligne à l'adresse suivante: http://www.picker-europe.de/index.php?publika tionen

\section{F. Des indications sur le personnel qui} a recueilli les données sont-elles mentionnées dans le rapport?

Une compétence sur le plan médical est nécessaire pour la saisie de certains indicateurs; pour d'autres, cela n'est pas une condition préalable.

Exemple: infection postopératoire de la plaie. Des connaissances médicales sont nécessaires pour évaluer si une plaie est infectée ou non. Cette estimation doit être réalisée par du personnel qualifié.

\section{G. La source des données est-elle décrite?}

Les données pour les rapports concernant la qualité peuvent provenir de diverses sources: banques de données internes à la clinique, dossiers médicaux, enquêtes auprès des patients, etc.

Exemple: les données concernant la fréquence de prescription de l'aspirine chez les patients victimes d'un infarctus du myocarde ont été relevées grâce à une enquête réalisée auprès de tous les patients à la sortie de l'hôpital.

\section{H. La durée de la mesure et de l'intervalle de temps sont-ils indiqués?}

Les mesures sont relevées pendant des périodes d'une durée variable, en fonction de l'indica- 
teur de qualité. Elles peuvent durer entre un jour et un an, ou plus longtemps encore.

Exemple: chez tous les patients victimes d'un infarctus du myocarde qui étaient hospitalisés du $1^{\text {er }}$ août au 31 octobre 2008.

I. Les données nécessaires à une éventuelle rectification de la composition du collectif des patients (casemix) ont-elles été recueillies? Lorsque les paramètres de résultats sont comparés entre différents hôpitaux ou cliniques, les résultats concernant la composition du collectif des patients doivent être corrigés, au moins en ce qui concerne les facteurs connus et qui peuvent être relevés. Il faudrait indiquer de manière détaillée dans le rapport, les données nécessaires (degré de gravité de la maladie, durée de la maladie, autres maladies, etc.) et mentionner les citations littéraires appropriées qui prouvent la pertinence et la justesse de la sélection des données recueillies en plus. Une correction n'est, en règle générale, pas nécessaire lors de la mesure de la qualité du processus.

Exemple: une étude comparative a été réalisée, entre différents hôpitaux en Suisse, sur la fréquence des patients atteints d'escarre survenue à l'hôpital. Les scores de Braden et Norton ont été utilisés pour la correction.

\section{J. La qualité des données relevées a-t-elle été contrôlée?}

Ce point concerne l'exhaustivité et la justesse des données. Comment a-t-on contrôlé si les données de tous les patients ont été correctement saisies?

Exemple: aspirine chez les patients victimes d'un infarctus du myocarde: tous les patients victimes d'un infarctus du myocarde ont été identifiés au sein du système de documentation interne à la clinique; on a ensuite vérifié dans chaque rapport médical si de l'aspirine avait bien été prescrite au patient. Une personne externe à l'hôpital a vérifié, par un contrôle aléatoire, si les données avaient été correctement relevées et introduites, comme il se doit, dans la banque de données.

K. Y-a-t-il des indications sur la manière dont on a procédé avec les données manquantes? Pour l'interprétation des résultats, il est important de savoir chez combien de patients les données étaient incomplètes ou impossibles à réunir. Les résultats doivent être interprétés avec prudence lorsque les données n'ont pas pu être relevées chez un grand nombre de patients. Des indications seraient nécessaires sur l'exhaustivité des données afin d'éviter les interprétations erronées.
Exemple: il n'était pas possible, chez 15\% des patients victimes d'un infarctus du myocarde, de clarifier si de l'aspirine leur avait été prescrite au moment de la sortie de l'hôpital. Plus la part de données manquantes est élevée, plus l'interprétation des résultats sera difficile. Il n'est pas possible d'indiquer une valeur limite à partir de laquelle les résultats ne peuvent plus être interprétés.

\section{La présentation des résultats est-elle} adéquate et intelligible?

Les résultats sont la plupart du temps indiqués en fréquences ou en pourcentages. Il faudrait éviter d'employer des termes incompréhensibles pour les profanes en médecine, comme par exemple le «rapport des chances» (ou odds ratio).

Exemples: $85 \%$ des patients victimes d'un infarctus du myocarde ont reçu, à leur sortie d'hôpital, une ordonnance pour de l'aspirine; ou: le taux de mortalité à 30 jours chez les patients victimes d'un infarctus du myocarde était de 6\% en 2007 (12 patients sur 200).

\section{La réalisation de la comparaison avec d'autres fournisseurs de prestations, est-elle décrite de manière détaillée?}

L'un des objectifs de la mesure de la qualité est la comparaison de la qualité de différents fournisseurs de prestations. Outre l'indicateur, il est donc nécessaire de relever des données supplémentaires (voir point I). La composition du collectif des patients (casemix) est corrigée sur la base de ces données et à l'aide de méthodes statistiques. Une justification plausible devrait accompagner l'absence de correction; lors de l'interprétation, il faudrait en outre signaler de manière explicite les limitations potentielles des résultats.

Les comparaisons entre les prestataires sont plus faciles à réaliser, lors de la mesure de la qualité du processus. Les résultats de différents hôpitaux peuvent être comparés et il est également possible de faire une comparaison avec une valeur cible définie ou avec des données publiées dans la littérature.

Exemple 1: par manque de données, aucune correction n'a été effectuée en ce qui concerne la composition du collectif des patients (casemix), lors de la comparaison, entre les hôpitaux suisses et allemands, du taux de mortalité suite à une attaque d'apoplexie. Les résultats doivent, de ce fait, être interprétés avec la plus grande précaution. Les différences prouvées ne peuvent pas simplement être expliquées par une qualité (meilleure ou moins bonne) des traitements; elles pourraient provenir de différences qui existent au niveau des collectifs de patients (âge, degré de gravité, autres maladies).

Exemple 2: l'objectif fixé par le canton X qui était de prescrire de l'aspirine à la sortie de l'hôpital à plus de $80 \%$ des patients souffrant d'un infarctus du myocarde, a été atteint par les hôpitaux $A, B$ et $C$; en revanche, les hôpitaux $D$, E et $F$ n'ont pas atteint cet objectif.

\section{N. Points d'ordre général}

Il faudrait également donner des indications sur les points suivants afin d'améliorer la transparence.

\section{a. Qui garantit la justesse des données}

et des interprétations?

Le rapport devrait indiquer de manière explicite, celui qui garantit la justesse des données. Comme pour les publications d'études cliniques, la personne qui effectue les analyses statistiques devrait être citée nommément et garantir leur justesse.

Exemple: le directeur de l'hôpital X garantit la justesse des données.

\section{b. Indications concernant le financement du relevé des données}

Dans la plupart des cas, le relevé des données est financé par l'institution responsable de l'hôpital considéré ou par un réseau de médecins.

Exemple: le relevé des données a été financé par l'hôpital X.

\section{c. La commission d'éthique a-t-elle donné,}

si nécessaire, son accord?

Les opinions divergent en ce qui concerne l'accord obligatoire d'une commission d'éthique et varient d'un canton à l'autre. C'est pourquoi il est recommandé de prendre contact avec les autorités cantonales compétentes et de se renseigner sur les détails, avant le relevé des données.

Exemple: l'accord des autorités compétentes a été obtenu pour le relevé des données décrites dans ce rapport.

\section{d. Les patients ont-ils donné, si nécessaire,} leur accord?

Les autorités cantonales compétentes peuvent décider si le consentement du patient est nécessaire pour le relevé des données. Dans certains cantons, les patients consignent dans un document, leur accord avec le relevé de données pour des projets concernant la qualité.

Exemple: par leur signature au moment de l'admission, les patients donnent leur accord au relevé des données. 
e. Qui est propriétaire des données recueillies?

Les données sont-elles accessibles

et peuvent-elles être examinées?

En règle générale, l'hôpital où sont relevées les données est propriétaire de ces dernières; elles devraient être stockées et consultables pendant un certain temps (il n'existe pas de règles précises à ce sujet).

Exemple: l'hôpital X est propriétaire des données publiées. Les données peuvent être consultées, dans les cas justifiés et avec le consentement de la direction de l'hôpital, auprès de l'instance chargée de la coordination de la qualité.

\section{f. A-t-on réalisé un audit externe} et indépendant des données recueillies? Les hôpitaux sont libres de faire contrôler par une instance externe l'authenticité de leurs données concernant la qualité.

Exemple: les données ont été contrôlées par les responsables de la qualité de l'hôpital; une évaluation externe n'a pas eu lieu.

\subsection{Intelligibilité}

Un commentaire d'accompagnement est souhaitable, étant donné que de nombreuses données ne sont pas faciles à interpréter pour les profanes en médecine: d'autre part, il convient d'éviter les interprétations erronées, dans l'intérêt de tous et pour les raisons mentionnées ci-dessus. Dans ce commentaire, les résultats devraient être décrits aux non-initiés de manière intelligible et les limitations propres aux nombres devraient faire l'objet d'explications détaillées.

Lors de l'élaboration d'un tel commentaire, il faut veiller, entre autres, aux points suivants:

Les résultats des mesures, représentés sous forme de nombres, sont-ils commentés? Ce commentaire doit exposer la conception de base relative à la qualité, les méthodes et les instruments employés et préciser la raison de ce choix. En outre, le commentaire devrait fournir une estimation fondée des résultats obtenus.

\section{Le langage employé et l'explication}

des méthodes statistiques sont-ils adaptés au public cible?

Lorsque le grand public est visé, le langage doit être approprié et les détails techniques nécessaires à la compréhension des nombres devraient être exposés de manière intelligible.

La documentation relative aux résultats présentés est-elle complète, détaillée et accessible?
Pour des raisons de transparence, les détails techniques de la collecte de données ou des modèles statistiques devraient être inclus dans une annexe librement accessible sur Internet, même lorsqu'ils ne sont pas contenus dans le rapport destiné aux médias ou au grand public. Les éventuelles publications scientifiques qui découlent de l'ouvrage concerné devraient être mentionnées.

\section{Les résultats sont-ils simplifiés, pour autant} que cela soit possible et judicieux?

Les résultats devraient être représentés le plus simplement possible, mais de manière correcte, afin d'être compris par le plus grand nombre de personnes (par ex. fréquence, pro-

\section{Checklist}

1. Pertinence

A. Les indicateurs choisis représentent-ils la qualité oui/non/en partie des traitements médicaux?

B. L'indicateur concerne-t-il la structure, un processus ou le résultat? structure/processus/ résultat

C. Le responsable du choix des indicateurs est-il mentionné? oui/non/en partie

2. Justesse

A. Les indicateurs sont-ils définis de manière précise? oui/non/en partie

B. La totalité des patients chez qui l'indicateur est mesuré, oui/non/en partie est-elle clairement décrite?

C. Existe-t-il des indications concrètes sur le nombre de patients chez lesquels l'indicateur a été relevé?

D. Dans le cas d'une enquête auprès des patients, existe-t-il des indications sur le taux de réponse?

E. Les instruments servant à la mesure des indicateurs sont-ils valides et fiables?

F. Des indications sur le personnel qui a recueilli les données sont-elles mentionnées dans le rapport?

G. La source des données est-elle décrite?

H. La durée de la mesure et de l'intervalle de temps sont-ils indiqués? oui/non/en partie

I. Les données nécessaires à une éventuelle rectification de la com- oui/non position du collectif des patients (casemix) ont-elles été recueillies?

J. La qualité des données relevées a-t-elle été contrôlée? oui/non/aucune indication

K. Y-a-t-il des indications sur la manière dont on a procédé avec oui/non les données manquantes?

L. La présentation des résultats est-elle adéquate et intelligible? oui/non/en partie

M. La réalisation de la comparaison avec d'autres fournisseurs oui/non/en partie de prestations est-elle décrite de manière détaillée?

$N$ Points d'ordre général

a. Qui garantit la justesse des données et des interprétations? indiqué/non indiqué

b. A-t-on fourni des indications concernant le financement du projet? oui/non

c. La commission d'éthique a-t-elle donné, si nécessaire, son accord? oui/non/pas nécessaire

d. Les patients ont-ils donné, si nécessaire, leur accord? oui/non/pas nécessaire

e. Qui est propriétaire des données recueillies? oui/non Les données sont-elles accessibles?

f. A-t-on réalisé un audit externe et indépendant oui/non des données recueillies?

\section{Intelligibilité}

Les résultats sont-ils présentés de façon intelligible et interprétés de manière compréhensible? oui/non/en partie 


\section{Existe-t-il une base de comparaison} pour les chiffres publiés?

Les données numériques gagnent en importance en présence d'une base de comparaison. Une telle base devrait être mise à disposition par l'auteur des analyses:

- Valeur de référence basée sur un consensus d'experts ou seuil de tolérance avec importance clinique (par ex., le degré de couverture vaccinale doit être supérieur à $90 \%$ pour atteindre une immunité de groupe).

- Résultats de l'année précédente des mêmes organisations (série chronologique).

- Répartition des résultats d'organisations participant à des études comparatives (quelquefois appelées benchmark): valeur moyenne et écart type, $25^{\mathrm{e}}, 50^{\mathrm{e}}$ et $75^{\mathrm{e}}$ percentiles, etc.

- Résultats publiés d'autres organisations, incluant les comparaisons internationales.

\section{L'incertitude des résultats est-elle évoquée?}

L'incertitude des résultats doit être évoquée lors de la publication: elle est la conséquence du nombre limité d'observations dans chaque organisation ou de l'inexactitude de la mesure. La manière de procéder est fonction du public cible. Les possibilités suivantes peuvent être envisagées:

- Indication des intervalles de confiance pour les résultats (en règle générale, une «marge d'erreur» est indiquée pour les sondages d'opinion).

- Indication d'une «différence statistiquement significative», lors de l'analyse de la valeur moyenne du groupe (ou d'une autre valeur de référence considérée comme importante).

- Commentaire libre (exemple: les résultats varient d'un hôpital à l'autre, mais il n'est pas exclu que la majorité de ces différences soit fortuite. Seul l'hôpital X présente des résultats sensiblement plus mauvais que la moyenne; cette différence ne peut pas être exclusivement imputée au hasard).

\section{Attire-t-on l'attention sur les avantages} et les inconvénients de la méthode de mesure? Chaque méthode de mesure présente ses avantages et ses inconvénients, ou plutôt ses limites. Les inconvénients et les limites doivent notamment être indiqués de manière explicite et faire l'objet d'un débat ouvert. Les objections justifiées à l'égard des résultats présentés devraient être anticipées dans le rapport Q.

\section{Checklist}

Lors de la publication des rapports concernant la qualité, leur auteur doit mentionner les cri- tères qui figurent sur cette liste et qui sont remplis. La transparence sur la manière dont les données ont été relevées et interprétées est ainsi possible.

\section{Annotations}

1 Ces recommandations ont été approuvées par le Sénat de l'Académie Suisse des Sciences Médicales le 19 mai 2009. Le groupe de travail qui a élaboré ces recommandations inclut les personnes suivantes: Prof. Johann Steurer, Zurich (président); Dr Francesca Giuliani, Schlieren; lic. oec. Pius Gyger, Zurich; Dr Daniel Herren, Zurich; Dr Olivier Kappeler, Weinfelden; Prof. Annemarie Kesselring, Berne; Margrit Kessler, Zurich; Prof. Urs Metzger, Zurich; Prof. Thomas Perneger, Genève; lic. rer. pol. Thomas Straubhaar, Berne; Dr Daniel Suter, Bâle; Dr Jürg von Below, Bienne; Dr Jean-Blaise Wasserfallen, Lausanne; Dr Heidemarie Weber, Bâle.

2 Dans ce texte, le genre masculin est utilisé pour faciliter la lecture; il va de soi qu'il est toujours question des deux genres.

3 Par publication, nous entendons la communication de données concernant la qualité, à des personnes et/ou des institutions qui ne s'étaient pas engagées de manière directe (médecin, département, clinique) ou indirecte (direction de l'hôpital, responsables de réseaux de médecins) lors du relevé des données concernant la qualité. Par communication, nous entendons par exemple la transmission de données concernant la qualité aux autorités politiques, aux caisses-maladie ou la publication dans les médias grand public.

4 Par qualité de l'indication, une forme spécifique de la qualité du processus, nous entendons l'adéquation (appropriateness) des prestations à fournir; celle-ci concerne par exemple la question de savoir si une coronarographie est indiquée ou non chez un patient atteint d'une angine de poitrine.

5 En application de l'article $321^{\text {bis }}$ du Code pénal suisse (CPS, RS 311.0); article 1, 3 alinéa 1,9 al. $5,10,11$ et 13 de l'ordonnance du 14 juin 1993.

$6 \quad$ Les chapitres 5.1. et 5.3. ont été rédigés par Hanspeter Kuhn, avocat, et par Gabriela Lang, avocate; tous deux font partie du service juridique de la FMH.

LAMal article 58 Garantie de la qualité

1 Après avoir consulté les organisations intéressées, le Conseil fédéral peut prévoir des contrôles scientifiques et systématiques pour garantir la qualité ou l'adéquation des prestations que l'assurance obligatoire des soins prend en charge.

2 Il peut en confier l'exécution aux associations professionnelles ou à d'autres institutions.

3 Il détermine les mesures servant à garantir ou à rétablir la qualité ou l'adéquation des prestations. Il peut en particulier prévoir que: a. l'accord du médecin-conseil est nécessaire avant l'exécution de certaines mesures diagnostiques ou thérapeutiques, notamment celles qui sont particulièrement coûteuses;

b. des mesures diagnostiques ou thérapeutiques particulièrement coûteuses ou difficiles ne seront prises en charge par l'assurance obligatoire des soins que lorsqu'elles sont pratiquées par des fournisseurs de prestations qualifiés en la matière. Il peut désigner ces fournisseurs de prestations.

OAMal article 77 Garantie de la qualité

1 Les fournisseurs de prestations ou leurs organisations élaborent des conceptions et des programmes en matière d'exigences de la qualité des prestations et de promotion de la qualité. Les modalités d'exécution (contrôle de l'observation, conséquences de l'inobservation, financement) sont réglées dans les conventions tarifaires ou dans des conventions particulières relatives à la garantie de la qualité conclues avec les assureurs ou leurs organisations. Ces réglementations doivent être conformes aux normes généralement reconnues, compte tenu du caractère économique des prestations.

2 Les parties à la convention sont tenues d'informer l'OFSP sur les clauses en vigueur. L'OFSP peut exiger un rapport sur l'application des règles de garantie de la qualité.

3 Dans les domaines où aucune convention n'a pu être conclue ou si la convention n'est pas conforme à l'al. 1, le Conseil fédéral édicte les dispositions nécessaires. Il consulte au préalable les organisations intéressées.

4 Le département détermine les mesures prévues à l'art. 58, al. 3 , de la loi, après consultation de la commission compétente.

8 LAMal article 22a Données des fournisseurs de prestations

1 Les fournisseurs de prestations doivent communiquer aux autorités fédérales compétentes les données qui sont nécessaires pour surveiller l'application des dispositions de la présente loi relatives au caractère économique et à la qualité des prestations. Les indications suivantes doivent notamment être communiquées: a. le genre d'activité, l'infrastructure et l'équipement, la forme juridique;

b. l'effectif du personnel et le nombre de places de formation ainsi que leur structure;

c. le nombre de patients et la structure de leur effectif, sous une forme anonyme;

d. le genre, l'ampleur et les coûts des prestations fournies;

e. les charges, les produits et le résultat d'exploitation;

f. les indicateurs de qualité médicaux.

2 Les personnes physiques ou morales interrogées sont soumises à l'obligation de renseigner. Les données doivent être mises gratuitement à disposition.

3 Les données sont collectées par l'Office fédéral de la statistique. Celui-ci met à la 
disposition de l'Office fédéral de la santé publique, du Surveillant fédéral des prix, de l'Office fédéral de la justice, des cantons et des assureurs ainsi que des institutions figurant à l'art. 84a, les données par fournisseur de prestations énumérées à l'al. 1 aux fins de l'application de la présente loi. Ces données sont publiées.

4 Le Conseil fédéral édicte des dispositions détaillées sur la collecte, le traitement, la transmission et la publication des données, dans le respect du principe de la proportionnalité.

9 Sont notamment publiés selon l'article 31 OAMal, version du 22 octobre 2008: a. offre de prestations des fournisseurs de prestations; b. diplômes et titres postgrades des fournisseurs de prestations; $c$. indicateurs $d e$ qualité médicaux; $\mathrm{d}$. étendue et genre des prestations fournies; e. évolution des coûts.

$10 \mathrm{Au}$ moment de sa déposition concernant une personne de l'histoire contemporaine suisse, le Professeur W. Hofer, historien reconnu, aurait été acquitté s'il avait vraiment été en mesure de s'appuyer sur un rapport du Tribunal cantonal de Zurich. cf. ATF 125 IV 298 (condamnation pénale du 4 novembre 1999), au motif qu'il n'aurait pas exclusivement dû se fonder sur la littérature secondaire, mais examiner lui-même les sources (c'est-à-dire le rapport).

11 Le chapitre 5.2. a été rédigé par le professeur Rolf $\mathrm{H}$. Weber (Prof. en droit des médias à l'Université de Zurich).

12 Sans être des indicateurs favorisés par le groupe de travail, les exemples permettent d'expliquer les critères pris séparément; en outre, ils ne prétendent pas à l'exhaustivité.
13 La validité d'un instrument indique s'il mesure réellement, grâce au test, la caractéristique qui est visée. Pour prendre un exemple: le questionnaire mesure-t-il réellement la satisfaction des patients ou recueille-t-il autre chose?

14 La «fiabilité» se rapporte à la fiabilité des mesures. À titre d'exemple, lorsque deux mesures sont effectuées à intervalle rapproché et dans des conditions identiques, les mesures réalisées à l'aide d'un instrument fiable produisent des résultats identiques ou au moins très similaires.

\section{Références}

1 Hibbard JH, Stockard J, Tusler M. Hospital performance reports: impact on quality, market share, and reputation. Health Aff (Millwood). 2005;24(4):1150-60.

2 Hibbard JH, Stockard J, Tusler M. Does publicizing hospital performance stimulate quality improvement efforts? Health Aff (Millwood). 2003;22(2):84-94.

3 Lindenauer PK, Remus D, Roman S, Rothberg MB, Benjamin EM, Ma A, et al. Public reporting and pay for performance in hospital quality improvement. N Engl J Med. 2007;356(5):486-96.

4 Werner RM, Asch DA. The unintended consequences of publicly reporting quality information. JAMA. 2005;293(10):1239-44.

5 Rehmann-Sutter C. Qualität messen oder Qualität bewerten. Schweiz Ärztezeitung. 2009;90:202-3.
6 Donabedian A. Evaluating the quality of medical care. Milbank Mem Fund Q. 1966;44(3):Suppl:166-206.

7 Tonstad S, Tonnesen P, Hajek P, Williams KE, Billing CB, Reeves KR. Effect of maintenance therapy with varenicline on smoking cessation: a randomized controlled trial. JAMA. 2006;296(1):64-71.

8 Lilford R, Mohammed MA, Spiegelhalter D, Thomson R. Use and misuse of process and outcome data in managing performance of acute medical care: avoiding institutional stigma. Lancet. 2004;363(9415):1147-54.

9 Rothberg MB, Morsi E, Benjamin EM, Pekow PS, Lindenauer PK. Choosing the best hospital: the limitations of public quality reporting. Health Aff (Millwood). 2008; 27(6):1680-7.

10 Housri N, Weil R, Shalowitz D, Koniaris LG. Should informed consent for cancer treatment include a discussion about hospital outcome disparities? PLOS Medicine. 2008;5(10):e 214.

11 Pronovost PJ, Berenholtz SM, Goeschel CA. Improving the quality of measurement and evaluation in quality improvement efforts. Am J Med Qual. 2008;23(2):143-6.

12 Pronovost PJ, Miller M, Wachter RM. The GAAP in quality measurement and reporting. JAMA. 2007;298(15):1800-2.

13 Dimick JB, Welch HG, Birkmeyer JD. Surgical mortality as an indicator of hospital quality: the problem with small sample size. JAMA. 2004;292(7):847-51. 